\title{
Serological survey of antibodies against peste des petits ruminants virus (pprv) and Mycoplasma capricolum subsp. Capripneumoniae (mccp) among small ruminants in northern Cameroon
}

\author{
Abubakar MB ${ }^{1 *}$, Baba SS ${ }^{1}$, Souley $\mathrm{HE}^{1,2}$, El-Yuguda AD ${ }^{1}$, Aboubakar $\mathrm{Y}^{2}$ and Zoyem $\mathrm{N}^{2}$ \\ ${ }^{1}$ Virology Research Laboratory, Department of Veterinary Microbiology, Faculty of Veterinary Medicine, University of Maiduguri, Nigeria \\ ${ }^{2}$ Laboratory National Veterinarian (LANAVET) Garou, Cameroon
}

\begin{abstract}
Serological survey to determine the prevalence of $M c c p$ and PPRV in small ruminants was carried out in Northern Cameroon. Sera from 500 animals comprising of 300 goats and 200 sheep were obtained from 131 flocks, within (13) localities and tested for the presence of antibodies against Mycoplsma capricolum subspecies capripneumoniae(Mccp) and peste des petits ruminants (PPRV) using blocking-enzyme linked immunosorbent assay (B-ELISA), compliment fixation test (CFT) and competitive enzymes linked immunosorbent assay (C-ELISA) respectively. A total of 50(16.7\%) and 30(10\%) goat sera tested were positive to $M c c p$ antigen using CFT and B-ELISA respectively and $73(24.3 \%)$ were positive to PPR-virus antigen using C-ELISA. While $83(41.5 \%)$ of the sheep sera reacted positively to PPRV antigen using C-ELISA and none of the sheep sera tested positive to Mccp using CFT. The prevalence was generally highest with PPRV infections only (46\%) followed by Mccp only (34\%) combined infection with Mccp and PPRV (14\%). However, no evidence of coinfection was found in sheep, the highest prevalence of infection (98\%) was noted to be PPRV only. In this study CFT was found to be more sensitive but less specific than B-ELISA in the detection of antibodies to Mccp. The use of C-ELISA for the detection of antibodies to PPRV was found to be more sensitive than the B-ELISA for detection of $M c c p$. The difference in the degree of homologous reaction to PPRV-N-Protein among the two species was statistically significant $(\mathrm{P}<0.05)$ was highest in sheep. Analysis of Geometrical mean titre (GMT) and mean percentage inhibition (MPI) of antibodies revealed consistently higher titre analysis of antibodies to PPRV (65) in goat and in sheep (80). Further analysis of serological profiles for the circulation of these respiratory pathogens revealed high values in locations closed and /or shared geographical boundary with sahelian zone of Nigeria and the period also the neighboring countries were these diseases are endermic also coincided with the peak harmattan period usually characterized by dry, dusty whether in this zone of the country. It is therefore suggested that cold dry, dusty climate of sudano-sahelian savannah could be a significant role as an extrinsic epidemiological determinant in the establishment of PPRV and Mccp infections among small ruminants population in northern province of Cameroon. There is therefore the need to embark on mass vaccination against PPRV. Also, the need to impose strict quarantine measures as well as the practice of testing and slaughter of animals imported from Mccp endermic areas into northern Cameroon for breeding, are suggested to prevent both introduction and further spread of this disease into the country.
\end{abstract}

\section{Introduction}

Small ruminants are very important domestic animal in tropical livestock production both for subsistence and economic development of the African continent. The subsistence sector pastoralist often depends on them for much of their livelihood [1,2]. They provide a flow of essential food products throughout the year, they also sustain the employment and income of millions of people in many rural areas of the world including Cameroon, where the small ruminants population in the far-north and Northern provinces were estimate to be $3,770,838$ representing $2,620,69.6$ goat and 1,150,142 sheep [3]. The two province have the highest population of small ruminant in the country representing $54.5 \%$ and $55.2 \%$ of goats and sheep in the far-north and $14.5 \%$ and $11.6 \%$ of goats and sheep respectively in the North Province [4].

In Cameroon, small ruminant is kept as source of capital [4]. They contribute energy and manure for Crop Production and are the only food and cash security available to many Africans [5]. In addition to their significant contribution to rural income, they contribute a substantial proportion of the nation's meat supply [6-8] as well as in ceremonial feasting. Annual report on meat and milk consumption per individual in Cameroon indicate an estimated of $12.2 \mathrm{~kg}$ and 11.6 litre of meat and milk consumption per annum respectively. Out of which goats and sheep meat account for $1.7 \mathrm{~kg}$ [4].

In spites of the economic importance of small ruminant a major constraint to their increased production in Africa has been the occurrence of infectious diseases, including respiratory disease in most part of the continent [9]. Among such disease are Mccp, PPRV and Pasteurella multocida $[4,7,8,10]$. Previous retrospective survey carried out in the study area have consistently revealed high mortality rates among small ruminants predominantly due to problems associated

*Correspondence to: Abubakar MB,Virology Research Laboratory, Department of Veterinary Microbiology, Faculty of Veterinary Medicine, University of Maiduguri, Nigeria; E-mail: mustydvm@gmail.com

Key words: peste des petites ruminantsvirus (PPRV); mycoplasma capricolum subsp., capripneumoniae; (Mccp), small ruminants; northern cameroon;

Received: April 10, 2017; Accepted: May 18, 2017; Published: May 22, 2017 
with pulmonary disease [4]. PPRV infection continues to be the most important single major cause of small ruminant disease with mortalities of over $50 \%$ [11-14].

In addition, analysis of specimens collected from slaughtered animal during routine examination at Garoua abattoir (in the north-province) yielded several respiratory pathogens including Mycoplasmascapricolum (Mcc), Pasteurella multocida and PPRV [15-17]. Mycoplasmas was reported to have been the major cause of economic losses in goat population in at least 30 countries in Africa and Asia containing a total goat population of more than 300 million $[18,19]$. The extent of the activity of these respiratory pathogens among small ruminant in the study area have not be adequately investigated, this study was designed to assess the prevalence of PPRV and Mccp infections among the small ruminant producing areas of far-north and north provinces of Cameroon, for better understanding of their effective control in the study areas.

\section{Materials and methods}

\section{Study area}

The study was carried out in thirteen (13) localities in the far-north and north province of Cameroon republic where the majority of the livestock population are owned and reared by nomads. Those locations in the north province include: Adoumri, Denbo, Jalingo, Kismatori, Nassaroa, Biddzar and Guilder there are characterized by a classic Sudanese tropical climate and the vegetation is made up of windy grassland, and sudano-sahelian savannah on the other hand the farnorth include: mayo-lone, Djamgliya, Koza and Mare are characterized by a tropical climate of moderate sahara type and the vegetation is mainly composed of thorny bush on sometimes rocky soils.

\section{Animal and sampling procedure}

A total of 500 blood samples were collected from 300 indigenes goats and 200 sheep from 131 flocks. Randomly selected from the two provinces (Northern and Far-north) in Cameroon republic. Samples were obtained by venipuncture from the every $5^{\text {th }}$ animal in a flock under semi intensive system of animal husbandry. The sera were separated from the clotted blood sample by centrifugation at $700 \mathrm{x} \mathrm{g}$ for ten minutes then preserved at $-20^{\circ} \mathrm{C}$ until tested.

Antigen control sera compliment and haemolytic system: the antigen used in this study include: inactivated Mccp derived from the reference F38 strain kindly supply by CIRAD-EMUT. Mouse monoclonal antibody (MAB) Mab "4.52" ascetic fluid against Mccp (The C-ELISA kit used in this procedure for the detection of specific anti PPRV antibody) was developed by CAMDA/OIE/EMVT and was kindly supplied by OIE. Commercially freeze-dried guinea pig $\mathrm{C}$ produced by Biomerieux ref. 72122 was used. The haemolytic system was prepared by adding equal volume of $3 \%$ meshed sieve nuts 1:700 diluted haemolytic serum.

\section{CFT}

The modified microtitre techniques described by O.I.E., 1996 was used in this work with minor modifications. $25 \mathrm{ml}$ of 2 folds dilution serum which has been decomplimented at $56^{\circ} \mathrm{C}$ for 30 minutes. Sample were treated against optimum dilution of the antigen and controlled individually. Readings were recorded at titres corresponding to the highest dilution that fixed $50 \%$ of C' i.e., $50 \%$ haemolysis was considered as positive.

\section{B-ELISA AND C-ELISA}

The Mccp B-ELISA was carried out as previously described by [20]. The test detects anti-Mcc antibody following addition of a mouse monoclonal antibody (MAB) into a test serum-Mcc is determined by the optical density values.

The PPRV C-ELISA described previously by [21] was used in this procedure. The test detects specific anti-PPRV antibody as the test serum depending on the binding of the Mab (the mouse Mab against PPR-virus-N protein) to a PPRV specific epitope in the presence of a positive serum. The test was performed in microtitre plate (NuncImmuno ${ }^{\mathrm{TM}}$ Maxisorp /cat: 439454) which were sensitized with PPRV reference antigen consisted of PPR virus- $\mathrm{N}$ protein produced in insect tissue culture cells infected with a recombinant baculovirus batch number "95-1".

\section{Data analysis}

The statistical differences between variables were analysed using the student ' $t$ ' test by pair wise comparison of variable. Were appropriate, the chi-square test and ANOVA were also employed for the test of statistical significance. They were all assessed at $\mathrm{P} \leq 0.05$ level of statistical significance.

\section{Result}

The result of the retrospective survey for the prevalence of antibodies against Mccp and PPRV in thirteen (13) localities in Northern Cameroon is summarized in Table 1.

Analysis of serological profiles for the presence of Contagious caprine pleuropnemoniae (CCPP) and peste des petits ruminants (PPR) showed comparable prevalence of antibody to the agents of the two diseases condition. In this study CFT (17\%) was found to be more sensitive but less specific than blocking- ELISA (10\%) in the detection of antibody to Mccp. Furthermore, the use of C-ELISA for the detection of antibodies to PPRV was found to be more sensitive than B-ELISA for detection of Mccp (Table 1). Analysis of the serological profile within sheep flock showed significantly higher prevalence of PPRV (42\%) than Mccp (0\%) among flocks studied. No antibody against Mccp was detected in the flock studied using CFT (Table 1).

Distribution of GMT and PMI of antibodies to PPRV and Mccp shows higher titre of antibodies to PPRV (65) in goats and in sheep (80) (Table 2) were consistently detected among small ruminants tested from Nassarao which compared favorably with the pattern of antibody to Mccp in the same location (Table 2). The analysis of mixed infections among goatwas significantly higher combine infection with Mccp and PPRV. The prevalence was generally highest with PPRV infection only (46\%) followed by Mccp only (34\%) and combined infection with PPRV and Mccp (14\%) (Table 3). However, no evidence of mixed infection was found is sheep; the highest prevalence of infection (98\%) was noted to be PPRV only (Table 4).

There was no significant difference $(\mathrm{P}>0.05)$ in the distribution of the total number of sheep and goat studied. However, significant difference $(\mathrm{p}<0.05)$ were noted between locations with the highest distributions observed among goats in Djaoli 65 (21.7\%) and Dembo $41(13.7 \%)$ similarly the highest number of sheep were studied in Guider 45(22.5\%) and Bokle 37(18.5\%) furthermore, significance sex difference $(\mathrm{p}<0.05)$ were also observe among the goat and sheep population studied. The number of male breed variations were generally observed among the sheep and goats studied. The number of female were consistently higher than those of male breed variations were generally 
Abubakar MB (2017) Serological survey of antibodies against peste des petits ruminants virus (pprv) and Mycoplasma capricolum subsp. Capripneumoniae(mccp) among small ruminants in northern Cameroon

Table 1. Serological test for demonstration of antibodies to PPRV and Mcc amomng small ruminants in Northern Cameroon

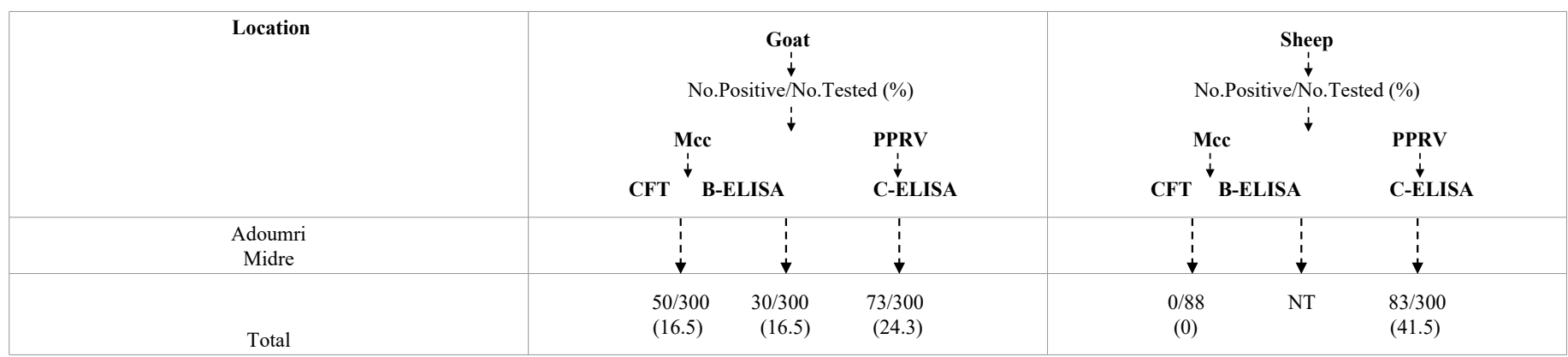

PPRV $=$ Peste de petiits ruminant virus

$\mathrm{MCC}=$ Mycoplasma Capricolum subsp. Capripnuemoniae

$\mathrm{CCPP}=$ Contagious caprine Pleuropneuminiae

NT $=$ Not tested

Table 2. Geometric mean titres, mean percentage inhibition of antibodies to respiratory pathogens in (Mccp, PPRV) small ruminnats in different locations studied.

\begin{tabular}{|c|c|c|c|c|c|}
\hline \multicolumn{3}{|c|}{ Goats } & \multicolumn{3}{|c|}{ Sheep } \\
\hline Location & PPRV & Mccp & & PPRV & Mccp \\
\hline & C-ELISA(MPI)* & CFT(GMT) & B-ELISA (MPI) & C-ELISA (MPI) & CFT(GMT)* \\
\hline Adounmri & 41.80 & 0 & 8.30 & 16.20 & 0 \\
\hline Bokle & 60.50 & 32 & 22.0 & 74.50 & 0 \\
\hline Dembo & 17.20 & 6.40 & 9.10 & 26.80 & 0 \\
\hline Djalingo & 49.20 & 8.0 & 11.0 & 44.80 & 0 \\
\hline Djaoli & 12.0 & 5.30 & 9.60 & 9.40 & 0 \\
\hline Kismatari & 52.0 & 4.0 & 11.40 & 60.40 & 0 \\
\hline Nassarao & 64.60 & 2.60 & 13.20 & 79.90 & 0 \\
\hline Bidzar & 13.80 & 4.80 & 10.20 & 12.90 & 0 \\
\hline Guider & 33.10 & 0 & 3.70 & 40.0 & 0 \\
\hline Mayo-loue & 40.20 & 4.0 & 6.50 & 24.30 & 0 \\
\hline Djingliya & 1.40 & 0 & 4.30 & 0.80 & 0 \\
\hline Koza & 10.80 & 0 & 3.90 & 3.80 & 0 \\
\hline Midre & 8.90 & 4.0 & 10.10 & 17.30 & 0 \\
\hline
\end{tabular}

B-ELISA $>20 \%=$ Positive

C-ELISA $>50 \%=$ Positive

CF-GMT $>1.4=$ Positive

GMT $=$ Geometric Mean Titre

*Geometric Mean Titre of Reciprocal of CF Antibody

MPl $=$ Mean Percentage Inhibition

Mccp $=$ Mycoplasma caprocolum subsp. capripneumoniae

$\mathrm{PPRV}=$ Peste des petites ruminants virus

Table 3. Evidence of mixed infections of respiratory pathogens in goats studied.

\begin{tabular}{|c|c|c|c|c|c|c|c|}
\hline \multicolumn{3}{|c|}{ Goats } & \multicolumn{5}{|c|}{ Number/ Total Number Positive (\%) } \\
\hline Location & Mccp/Mycoplasmosis only & PPRV only & $\begin{array}{c}\text { Pasteurella } \\
\text { infection only }\end{array}$ & Mccp + PPRV & $\begin{array}{l}\text { Mccp+ Pateurella } \\
\text { infection }\end{array}$ & $\begin{array}{c}\text { PPRV + Pasteurella } \\
\text { infection }\end{array}$ & $\begin{array}{c}\text { Mccp + Pasteurella } \\
\text { + PPRV }\end{array}$ \\
\hline Adounmri & $0 / 6 /(10)$ & $5 / 6(83.3)$ & $1 / 6(16.67)$ & $0 / 6(0)$ & $0 / 6(0)$ & $0 / 6(0)$ & $0 / 6(0)$ \\
\hline Bokle & $0 / 2(0)$ & $1 / 2(50)$ & $0 / 2(0)$ & $1 / 2(50)$ & $0 / 2(0)$ & $0 / 2(0)$ & $0 / 2(0)$ \\
\hline Dembo & $5 / 13(38.5)$ & $4 / 13(30.8)$ & $0 / 13(0)$ & $3 / 13(23.1)$ & $0 / 13(0)$ & $0 / 13(0)$ & $0 / 13(7.7)$ \\
\hline Djalingo & $1 / 5(20)$ & $3 / 5(60.0)$ & $0 / 5(0)$ & $0 / 20(0)$ & $0 / 5(0)$ & $0 / 5(0)$ & $0 / 5(0)$ \\
\hline Djaoli & $15 / 20(75)$ & $0 / 20(0)$ & $4 / 20(20.0)$ & $0 / 20(0)$ & $1 / 20(5)$ & $1 / 20(5)$ & $1 / 20(0)$ \\
\hline Kismatari & $8 / 22(36.4)$ & $7 / 22(31.8)$ & $0 / 22(0)$ & $7 / 22(40.0)$ & $0 / 22(0)$ & $0 / 22(0)$ & $0 / 22(0)$ \\
\hline Nassarao & $1 / 10(10)$ & $5 / 10(50)$ & $0 / 10(0)$ & $4(10(40.0)$ & $0 / 10(0)$ & $0 / 10(0)$ & $0 / 100(0)$ \\
\hline Bidzar & $4 / 6(66.7)$ & $2 / 6(33.4)$ & $0 / 6(0)$ & $0 / 6(0)$ & $0 / 6(0)$ & $0 / 6(0)$ & $0 / 6(0)$ \\
\hline Guider & $0 / 13(0)$ & 13/13(100) & $0 / 13(0)$ & $0 / 13(0)$ & $0 / 13(0)$ & $0 / 13(0)$ & $0 / 13(0)$ \\
\hline Mayo-loue & $2 / 13(15.4)$ & 11/13(84.6) & $0 / 13(0)$ & 0 & 0 & 0 & 0 \\
\hline Djingliya & 0 & 0 & 0 & 0 & 0 & 0 & 0 \\
\hline Koza & $0 / 4(0)$ & $4 / 4(100)$ & $0 / 4(0)$ & $0 / 4(0)$ & $0 / 4(0)$ & $0 / 4(0)$ & $0 / 4(0)$ \\
\hline Midre & $5 / 6(83.4)$ & $0 / 60(0)$ & $0 / 6(0)$ & $1 / 6(16.7)$ & $0 / 60(0)$ & $0 / 60(0)$ & $0 / 60(0)$ \\
\hline Total & $41 / 120(34.2)$ & $55 / 120(45.8)$ & $5 / 12(4.2)$ & $17 / 120(14.2)$ & $1 / 120(0.8)$ & $0 / 120(0)$. & $1 / 120(0.8)$ \\
\hline
\end{tabular}


Abubakar MB (2017) Serological survey of antibodies against peste des petits ruminants virus (pprv) and Mycoplasma capricolum subsp. Capripneumoniae(mccp) among small ruminants in northern Cameroon

Table 4. Evidence of mixed infections of respiratory pathogens in sheep studied.

\begin{tabular}{|c|c|c|c|c|c|c|c|}
\hline \multicolumn{3}{|c|}{ Goats } & \multicolumn{5}{|c|}{ Number/ Total Number Positive (\%) } \\
\hline Location & Mccp/Mycoplasmosis only & PPRV only & $\begin{array}{c}\text { Psteurella infection } \\
\text { only }\end{array}$ & Mccp + PPRV & $\begin{array}{l}\text { Mccp + Pateurella } \\
\text { infection }\end{array}$ & $\begin{array}{c}\text { PPRV + Pasteurella } \\
\text { infection }\end{array}$ & $\begin{array}{c}\text { Mccp + Pasteurella } \\
\text { + PPRV }\end{array}$ \\
\hline Adounmri & $0 / 2(0)$ & $1 / 2(50)$ & $1 / 2(50)$ & $0 / 2(0)$ & $0 / 2(0)$ & $0 / 2(0)$ & $0 / 2(0)$ \\
\hline Bokle & $0 / 36(0)$ & $36 / 36(100)$ & $36 / 36(0)$ & $0 / 36(0)$ & $0 / 36(0)$ & $0 / 36(0)$ & $0 / 36(0)$ \\
\hline Dembo & $0 / 2(0)$ & $1 / 2(50)$ & $1 / 2(50)$ & $00 / 2(0)$ & $00 / 2(0)$ & $00 / 2(0)$ & $00 / 2(0)$ \\
\hline Djalingo & $0 / 3(0)$ & $3 / 3(100)$ & $3 / 3(0)$ & $0 / 3(0)$ & $0 / 3(0)$ & $0 / 3(0)$ & $0 / 3(0)$ \\
\hline Djaoli & 0 & 0 & 0 & 0 & 0 & 0 & 0 \\
\hline Kismatari & $0 / 10(0)$ & $10 / 10(100)$ & $10 / 10(0)$ & $0 / 10(0)$ & $0 / 10(0)$ & $0 / 10(0)$ & $0 / 10(0)$ \\
\hline Nassarao & $0 / 6(0)$ & $6 / 6(100)$ & $6 / 6(0)$ & $0 / 60(0)$ & $0 / 6(0)$ & $0 / 6(0)$ & $0 / 6(0)$ \\
\hline Bidzar & $0 / 1(0)$ & $1 / 1(100)$ & $1 / 60(0)$ & $0 / 1(0)$ & $0 / 1(0)$ & $0 / 1(0)$ & $0 / 1(0)$ \\
\hline Guider & $0 / 20(0)$ & $20 / 20(100)$ & $0 / 20(0)$ & $0 / 20(0)$ & $0 / 20(0)$ & $0 / 20(0)$ & $0 / 20(0)$ \\
\hline Mayo-loue & $0 / 1 /(0)$ & $1 / 1(100)$ & $0 / 1(0)$ & $0 / 1(0)$ & $0 / 1(0)$ & $0 / 1(0)$ & $0 / 1(0)$ \\
\hline Djingliya & 0 & 0 & 0 & 0 & 0 & 0 & 0 \\
\hline Koza & $0 / 10(0)$ & $1 / 1(100)$ & $0 / 1(0)$ & $0 / 1(0)$ & $0 / 1(0)$ & $0 / 1(0)$ & $0 / 1(0)$ \\
\hline Midre & $0 / 3(0)$ & $3 / 3(100)$ & $0 / 3(0)$ & $0 / 3(0)$ & $0 / 3(0)$ & $0 / 3(0)$ & $0 / 3(0)$ \\
\hline Total & $0 / 85(0)$ & $83 / 85(97.7)$ & $2 / 85(2.4)$ & $0 / 85(0)$ & $0 / 85(0)$ & $0 / 85(0)$ & $0 / 85(0)$ \\
\hline
\end{tabular}

Table 5. Sex and breed distribution of small ruminants in different locations studied .

\begin{tabular}{|c|c|c|c|c|c|c|c|c|c|c|c|c|c|c|c|c|c|}
\hline \multirow{3}{*}{\begin{tabular}{|l|}
\multicolumn{1}{|c}{ Location } \\
Location \\
\end{tabular}} & \multicolumn{4}{|c|}{$\operatorname{Sex}(\%)$} & \multicolumn{9}{|c|}{ Breed (\%) } & \multicolumn{4}{|c|}{ Other Species Total (\%) } \\
\hline & \multicolumn{3}{|c|}{ Goats } & \multicolumn{4}{|c|}{ Sheep } & \multicolumn{3}{|c|}{ Goats } & \multicolumn{7}{|c|}{ Sheep } \\
\hline & Male & Female & Total & Male & Female & Total & Kirdi & Bororo & Total & Kirdi & Foulbe & Ouda & Total & Cattle & equine & swine & $\begin{array}{c}\text { Avina } \\
\text { sp. }\end{array}$ \\
\hline Adounmri & $\begin{array}{c}1 \\
(10)\end{array}$ & $\begin{array}{c}9 \\
(90)\end{array}$ & 10 & $\begin{array}{c}2 \\
(28.6)\end{array}$ & $\begin{array}{c}5 \\
(71.4)\end{array}$ & 7 & $\begin{array}{c}10 \\
(100)\end{array}$ & $\begin{array}{c}0 \\
(0)\end{array}$ & 10 & $\begin{array}{c}7 \\
(100)\end{array}$ & $\begin{array}{c}0 \\
(10)\end{array}$ & $\begin{array}{c}0 \\
(0)\end{array}$ & 7 & $\begin{array}{c}0 \\
(0)\end{array}$ & $\begin{array}{c}1 \\
(11.1)\end{array}$ & $\begin{array}{c}0 \\
(0)\end{array}$ & $\begin{array}{c}37 \\
(3.8)\end{array}$ \\
\hline Bokle & $\begin{array}{c}0 \\
(0)\end{array}$ & $\begin{array}{c}2 \\
(100)\end{array}$ & 2 & $\begin{array}{c}3 \\
(8.1)\end{array}$ & $\begin{array}{c}34 \\
(92.0)\end{array}$ & 37 & $\begin{array}{c}2 \\
(100)\end{array}$ & $\begin{array}{c}0 \\
(0)\end{array}$ & 2 & $\begin{array}{c}6 \\
(16.2)\end{array}$ & $\begin{array}{c}31 \\
(83.8)\end{array}$ & $\begin{array}{c}0 \\
(0)\end{array}$ & 37 & $\begin{array}{c}218 \\
(17.8)\end{array}$ & $\begin{array}{c}3 \\
(33.3)\end{array}$ & $\begin{array}{c}10 \\
(45.5)\end{array}$ & $\begin{array}{c}0 \\
(0)\end{array}$ \\
\hline Dembo & $\begin{array}{c}6 \\
(14.6)\end{array}$ & $\begin{array}{c}35 \\
(85.4)\end{array}$ & 41 & $\begin{array}{c}2 \\
(40)\end{array}$ & $\begin{array}{c}3 \\
(60)\end{array}$ & 5 & $\begin{array}{c}35 \\
(85.4)\end{array}$ & $\begin{array}{c}6 \\
(14.6)\end{array}$ & 41 & $\begin{array}{c}5 \\
(100)\end{array}$ & $\begin{array}{c}0 \\
(0)\end{array}$ & $\begin{array}{c}0 \\
(0)\end{array}$ & 5 & $\begin{array}{c}63 \\
(5.1)\end{array}$ & $\begin{array}{c}0 \\
(0)\end{array}$ & $\begin{array}{c}0 \\
(0)\end{array}$ & $\begin{array}{c}141 \\
(14.4)\end{array}$ \\
\hline Djalingo & $\begin{array}{c}3 \\
(33.3)\end{array}$ & $\begin{array}{c}6 \\
(66.7)\end{array}$ & 9 & $\begin{array}{c}1 \\
(25)\end{array}$ & $\begin{array}{c}3 \\
(75)\end{array}$ & 4 & $\begin{array}{c}9 \\
(100)\end{array}$ & $\begin{array}{c}0 \\
(0)\end{array}$ & 9 & $\begin{array}{c}4 \\
(100)\end{array}$ & $\begin{array}{c}0 \\
(0)\end{array}$ & $\begin{array}{c}0 \\
(0)\end{array}$ & 4 & $\begin{array}{c}19 \\
(1.6)\end{array}$ & $\begin{array}{c}0 \\
(0)\end{array}$ & $\begin{array}{c}0 \\
(0)\end{array}$ & $\begin{array}{c}13 \\
(1.3)\end{array}$ \\
\hline Djaoli & $\begin{array}{c}6 \\
(9.2)\end{array}$ & $\begin{array}{c}59 \\
(90.8)\end{array}$ & 65 & $\begin{array}{c}0 \\
(0)\end{array}$ & $\begin{array}{c}15 \\
(100)\end{array}$ & 15 & $\begin{array}{c}65 \\
(100)\end{array}$ & $\begin{array}{c}0 \\
(0)\end{array}$ & 65 & $\begin{array}{c}15 \\
(100)\end{array}$ & $\begin{array}{c}0 \\
(0)\end{array}$ & $\begin{array}{c}0 \\
(0)\end{array}$ & 15 & $\begin{array}{l}86 \\
(0)\end{array}$ & $\begin{array}{c}0 \\
(0)\end{array}$ & $\begin{array}{c}0 \\
(0)\end{array}$ & $\begin{array}{c}0 \\
(0)\end{array}$ \\
\hline Kismatari & $\begin{array}{c}5 \\
(20) \\
\end{array}$ & $\begin{array}{c}20 \\
(80)\end{array}$ & 25 & $\begin{array}{c}1 \\
(8.3)\end{array}$ & $\begin{array}{c}11 \\
(91.7)\end{array}$ & 12 & $\begin{array}{c}24 \\
(100)\end{array}$ & $\begin{array}{c}0 \\
(0)\end{array}$ & 25 & $\begin{array}{c}8 \\
(66.7)\end{array}$ & $\begin{array}{c}4 \\
(33.3)\end{array}$ & $\begin{array}{c}0 \\
(0)\end{array}$ & 12 & $\begin{array}{c}25 \\
(2.0)\end{array}$ & $\begin{array}{c}0 \\
(0)\end{array}$ & $\begin{array}{c}0 \\
(0)\end{array}$ & $\begin{array}{c}0 \\
(0)\end{array}$ \\
\hline Nassarao & $\begin{array}{c}6 \\
(46.2)\end{array}$ & $\begin{array}{c}7 \\
(53.8)\end{array}$ & 13 & $\begin{array}{c}2 \\
(28.6)\end{array}$ & $\begin{array}{c}5 \\
(71.4)\end{array}$ & 7 & $\begin{array}{c}13 \\
(100)\end{array}$ & $\begin{array}{c}0 \\
(0)\end{array}$ & 13 & $\begin{array}{c}7 \\
(100)\end{array}$ & $\begin{array}{c}0 \\
(0)\end{array}$ & $\begin{array}{c}14 \\
(100)\end{array}$ & 7 & $\begin{array}{c}0 \\
(0)\end{array}$ & $\begin{array}{c}0 \\
(0)\end{array}$ & $\begin{array}{c}0 \\
(0)\end{array}$ & $\begin{array}{c}0 \\
(0)\end{array}$ \\
\hline Bidzar & $\begin{array}{c}8 \\
(30.8)\end{array}$ & $\begin{array}{c}18 \\
(69.2)\end{array}$ & 26 & $\begin{array}{c}4 \\
(28.6)\end{array}$ & $\begin{array}{c}10 \\
(71.4)\end{array}$ & 14 & $\begin{array}{c}26 \\
(100)\end{array}$ & $\begin{array}{c}0 \\
(0)\end{array}$ & 26 & $\begin{array}{c}0 \\
(0)\end{array}$ & $\begin{array}{c}0 \\
(0)\end{array}$ & $\begin{array}{c}4 \\
(8.9)\end{array}$ & 14 & $\begin{array}{c}355 \\
(28.9)\end{array}$ & $\begin{array}{c}0 \\
(0)\end{array}$ & $\begin{array}{c}0 \\
(0)\end{array}$ & $\begin{array}{c}118 \\
(12.0)\end{array}$ \\
\hline Guider & $\begin{array}{c}10 \\
(38.5)\end{array}$ & $\begin{array}{c}16 \\
(61.5)\end{array}$ & 26 & $\begin{array}{c}8 \\
(17.8)\end{array}$ & $\begin{array}{c}37 \\
(82.2)\end{array}$ & 45 & $\begin{array}{c}26 \\
(100)\end{array}$ & $\begin{array}{c}0 \\
(0)\end{array}$ & 26 & $\begin{array}{c}10 \\
(22.2)\end{array}$ & $\begin{array}{c}31 \\
(68.9)\end{array}$ & $\begin{array}{c}1 \\
(25)\end{array}$ & 45 & $\begin{array}{c}445 \\
(36.2)\end{array}$ & $\begin{array}{c}0 \\
(0)\end{array}$ & $\begin{array}{c}0 \\
(0)\end{array}$ & $\begin{array}{c}209 \\
(21.3)\end{array}$ \\
\hline Mayo-loue & $\begin{array}{c}7 \\
(25)\end{array}$ & $\begin{array}{c}21 \\
(75)\end{array}$ & 28 & $\begin{array}{c}2 \\
(50)\end{array}$ & $\begin{array}{c}2 \\
(50)\end{array}$ & 4 & $\begin{array}{c}28 \\
(100)\end{array}$ & $\begin{array}{c}0 \\
(0)\end{array}$ & 28 & $\begin{array}{c}3 \\
(75)\end{array}$ & $\begin{array}{c}0 \\
(0)\end{array}$ & $\begin{array}{c}0 \\
(0)\end{array}$ & 4 & $\begin{array}{c}12 \\
(1.0)\end{array}$ & $\begin{array}{c}5 \\
(55.6)\end{array}$ & $\begin{array}{c}0 \\
(0)\end{array}$ & $\begin{array}{c}253 \\
(25.8)\end{array}$ \\
\hline Djingliya & $\begin{array}{c}0 \\
(0)\end{array}$ & $\begin{array}{c}3 \\
(100)\end{array}$ & 3 & $\begin{array}{c}1 \\
(11.1)\end{array}$ & $\begin{array}{c}8 \\
(88.5)\end{array}$ & 9 & $\begin{array}{c}3 \\
(100)\end{array}$ & $\begin{array}{c}0 \\
(0)\end{array}$ & 3 & $\begin{array}{c}9 \\
(100)\end{array}$ & $\begin{array}{c}0 \\
(0)\end{array}$ & $\begin{array}{c}0 \\
(0)\end{array}$ & 9 & $\begin{array}{c}0 \\
(0)\end{array}$ & $\begin{array}{c}0 \\
(0)\end{array}$ & $\begin{array}{c}0 \\
(0)\end{array}$ & $\begin{array}{c}0 \\
(0)\end{array}$ \\
\hline Koza & $\begin{array}{c}5 \\
(17.9)\end{array}$ & $\begin{array}{c}23 \\
(82.1)\end{array}$ & 28 & $\begin{array}{c}3 \\
(11.5)\end{array}$ & $\begin{array}{c}23 \\
(88.5)\end{array}$ & 26 & $\begin{array}{c}28 \\
(100)\end{array}$ & $\begin{array}{c}0 \\
(0)\end{array}$ & 28 & $\begin{array}{c}26 \\
(100)\end{array}$ & $\begin{array}{c}0 \\
(0)\end{array}$ & $\begin{array}{c}0 \\
(0)\end{array}$ & 26 & $\begin{array}{c}2 \\
(0.2)\end{array}$ & $\begin{array}{c}0 \\
(0)\end{array}$ & $\begin{array}{c}0 \\
(0)\end{array}$ & $\begin{array}{c}150 \\
(15.3)\end{array}$ \\
\hline Midre & $\begin{array}{c}11 \\
(25.8)\end{array}$ & $\begin{array}{c}13 \\
(54.2)\end{array}$ & 24 & $\begin{array}{c}3 \\
(20)\end{array}$ & $\begin{array}{c}12 \\
(80)\end{array}$ & 15 & $\begin{array}{c}24 \\
(100)\end{array}$ & $\begin{array}{c}0 \\
(0)\end{array}$ & 24 & $\begin{array}{c}15 \\
(100)\end{array}$ & $\begin{array}{c}0 \\
(0)\end{array}$ & $\begin{array}{c}0 \\
(0)\end{array}$ & 15 & $\begin{array}{c}3 \\
(0.2)\end{array}$ & $\begin{array}{c}0 \\
(0)\end{array}$ & $\begin{array}{c}12 \\
(54.5)\end{array}$ & $\begin{array}{c}60 \\
(6.1)\end{array}$ \\
\hline Total & $\begin{array}{c}68 \\
(22.7)\end{array}$ & $\begin{array}{c}232 \\
(77.3)\end{array}$ & 300 & $\begin{array}{c}32 \\
(16.0)\end{array}$ & $\begin{array}{c}168 \\
(84.0)\end{array}$ & 200 & $\begin{array}{c}294 \\
(98.0)\end{array}$ & $\begin{array}{c}6 \\
(2.0)\end{array}$ & 300 & $\begin{array}{c}115 \\
(57.5)\end{array}$ & $\begin{array}{c}66 \\
(33.5)\end{array}$ & $\begin{array}{c}19 \\
(9.5)\end{array}$ & 200 & $\begin{array}{c}1228 \\
(54.8)\end{array}$ & $\begin{array}{c}9 \\
(0.4)\end{array}$ & $\begin{array}{c}22 \\
(1.0\end{array}$ & $\begin{array}{c}981 \\
(43.8)\end{array}$ \\
\hline
\end{tabular}

observed among the sheep and goats studied. The 'Kirdi' breed were consistently more in number $409(82 \%)$ than other breeds (Table 5). The age distribution of the animal studied ranged from months and above (age testes) no significant difference ( $p>0.05)$ in the distribution of different age groups of sheep and goats studied. However, significant difference $(\mathrm{p}<0.05)$ were noted in the age distribution among different locations in Guider animal in the age group amount to 3 years were more in number $22(85 \%)$ than those greater than 3 years $4(14 \%)$. However, there was no significant difference $(p>0.05)$ in the age distribution of sheep in different locations (Table 6).

\section{Discussion}

Seroepidemiological investigations carried out in the present study using different serological methods have revealed considerable activity of respiratory pathogens of CCPP and PPR. However, significant differences were observed in the relative sensitivities and specificities of the serological method used. For instance, CFT (17\%) was found to be more sensitive but less specific than B-ELISA (10\%) in the detection of antibody to Mccp. This observation seems to be inconsistent with previous efforts on the comparative sensitivity and 
Abubakar MB (2017) Serological survey of antibodies against peste des petits ruminants virus (pprv) and Mycoplasma capricolum subsp. Capripneumoniae(mccp) among small ruminants in northern Cameroon

Table 6. Age distribution of small ruminants in different locations studies

\begin{tabular}{|c|c|c|c|c|c|c|}
\hline \multicolumn{4}{|c|}{ Goats } & \multirow{2}{*}{\multicolumn{3}{|c|}{$\begin{array}{c}\text { Sheep } \\
\text { Age group (\%) }\end{array}$}} \\
\hline \multirow[t]{2}{*}{ Location } & \multicolumn{3}{|c|}{ Age group (\%) } & & & \\
\hline & 9 months -3 years & $>3$ years & Total & 9 months -3 years & $>3$ years & Total \\
\hline Adounmri & $4(40)$ & $6(60)$ & 10 & $4(57.1)$ & $3(42.9)$ & 7 \\
\hline Bokle & $2(100)$ & $0(0)$ & 2 & $18(48.7)$ & $19(51.4)$ & 37 \\
\hline Dembo & $24(58.5)$ & $17(41.5)$ & 41 & $1(20.0)$ & $4(80.0)$ & 5 \\
\hline Djalingo & $4(44.4)$ & $5(55.5)$ & 9 & $2(50.0)$ & $2(50.0)$ & 4 \\
\hline Djaoli & $35(53.8)$ & $30(46.2)$ & 65 & $11(73.3)$ & $4(26.7)$ & 15 \\
\hline Kismatari & $14(56)$ & $11(44)$ & 25 & $8(66.7)$ & $4(33.3)$ & 12 \\
\hline Nassarao & $7(53.8)$ & $6(46.2)$ & 13 & $2(28.6)$ & $5(71.4)$ & 7 \\
\hline Bidzar & $16 / 61.5)$ & $10(38.5)$ & 26 & $5(35.7)$ & $9(64.3)$ & 14 \\
\hline Guider & $22(84.6)$ & $4(15.4)$ & 26 & $17(376.8)$ & $28(62.2)$ & 45 \\
\hline Mayo-loue & $18(64.3)$ & $10(35.7)$ & 28 & $3(75.0)$ & $1(25.0)$ & 4 \\
\hline Djingliya & $1(33.3)$ & $2(66.7)$ & 3 & $6(66.7)$ & $3(33.3)$ & 9 \\
\hline Koza & $13(46.4)$ & $15(53.6)$ & 28 & $16(61.5)$ & $10(38.5)$ & 26 \\
\hline Midre & $15(62.5)$ & $9(37.5)$ & 24 & $10(66.7)$ & $5(33.3)$ & 15 \\
\hline Total & $175(58.3)$ & $125(41.7)$ & 300 & $103(51.5)$ & $97(48.5)$ & 200 \\
\hline
\end{tabular}

specificity of CFT and ELISA techniques in serological analysis as it had been demonstrated by previous workers [22]. Generally, this study has revealed a considerable activity of PPRV among the different age groups of small ruminants in northern Cameroon. These results confirm the earlier findings which indicate the high endermicity of PPR in the study areas [7,8,13,16,23-25]. In addition, this study has also shown a higher prevalence of PPR in sheep than goats which is contrary to some previous reports $[7,26-28]$. Since the disease has been well documented $[10,25,26,29]$. The present findings could be as a result of various factors, the period of study, location and breed of small ruminants studied.

There is need to embark on a mass vaccination programme against PPR in both species. TCRV has been reported to protect small ruminants against PPRV[7,10,11,30,31]. However, the homologous vaccine against the disease was found to be more effective[23], although yearly booster vaccination may be required in order to ensure long lasting protective immunity against the PPRV infection. Both vaccines are produced commercially at LANAVET Cameroon and commonly named Bovipestrovax and Capripestovax respectively.

Furthermore, the present study has also revealed for the first time the occurrence of CCPP in Cameroon due to the detection of antibodies against Mycoplasma capricolum subspecies Capripneumoniae (Mccp) the goat sera examined during the study. A serological survey carried out previously in the northern Cameroon had failed to sufficiently established the presence of Mccp in the study areas [15,16]. This study however had to be belief that Mccp might have been long existed in the study areas. Since introduction of new flock was not a common practice among the farmers in the study area [32]. This therefore suggest that there is high tendency to incriminate the uncontrolled animal movement between northern Cameroon and the neighboring countries in the east and west primarily with those in the east Africa were CCPP is common and Mccp has been isolate in Tchad as partly responsible for the high mortality in the study area $[15,20,33]$.

The present study has provided preliminary data for further investigation into prevalence of CCPP in northern Cameroon including future work on serosurvelliance and attempt to isolate Mccp so as to be adequately characterized and their epidemiological role be defined as a respiratory pathogen of small ruminant in Cameroon. Beside testing and slaughtering of positive animal should be enforced to prevent further of the disease into the other regions.

\section{Acknowledgement}

The authors acknowledge with thanks the technical and editorial assistance of Drs A. Souley, T. Thiaucourt, D. Diallo, N. Andre, N. Zoyem of LANAVET Cameroon. The senior author was an undergraduate research project student in this Virus Research Laboratory University of Maiduguri.

\section{References}

1. Devendra C, McLeroy GB (1982) Introduction in: Goats and sheep production in the tropics. Longman, U.K. Ltd. 1-8.

2. Assim A, Rashid A, Chaudhary AH, Noor MS (2009) Production of homologous live attenuated cell culture vaccine for the control of Peste des Petits Ruminants in small ruminants. J Pak Vet 29: 72-74.

3. Bulteau P, Barazandeh D, Njoya A, Zakariaou B, Koulandi J (1998) Etude de faisabilite d'un project de rehabilitation des pointsd'eau pour le betaildans les provinces du Nord et de 1'extreme-Nord au Cameroun. 110pp.

4. Planchenault D (1993) Enquete de productive au Cameroun.Rapport final. MaisonsAlfort, France, CIRAD-EMVT, Pp. 249.

5. Brumby P (1990) Systems and livestock development in tropical Africa. In: Dauda $\mathrm{K}$ Jawara(ed), Animal disease as a factor limiting economic development in Africa. Cornell Veterinarian 80: 17-25.

6. Anon (1987) The sixth 5 year economic, social and development plan, 1987-1991.7th edition, ministry of plan and regional management (MINPAT), Yaounde, Cameroon.

7. Saliki JT, Ekue FN, Tanya VN, Douffissa A (1987) Peste des petits ruminants in Cameroun: A preliminary report. Rev Sci et Tech Ser Agrom3: 153-159.

8. Ekue NF, Tanya VN, Ndi C, Saliki JT (1992) A serological survey of antibodies against peste des petits ruminants (PPR) virus in small ruminnats in Cameroon. Bull AnimHlth Prod Afr 40: 49-53.

9. Egwu GO (1995) The application of Biotechnology in the diagnosis of Contagious Bovine Pleuropneumonia (CBPP) caused by Mycoplasma mycoidessubspecies mycoides SC: A member of mycoides cluster. Faculty of Veterinary Medicine, University of Maiduguri, Borno state. Nigeria. Seminar papers, 1: 1-3.

10. Fakri F, Ghzal F, Daouam S, ElarkamA, DouiebL, Zouheir Y, et al. (2015) Development and field application of a new combined vaccine against Peste des Petits Ruminants and Sheep Pox. Trials in Vaccinology4: 33-37.

11. Majiyagbe KA, Shamaki D, Kulu D, Udeani TKC (1992) Peste des petits ruminants (PPR) and Rinderpest (RP) antibodies in clinically normal small ruminants in Cameroon and Nigeria. Small Ruminant Research and Development in Africa. Proceedings of the second Biennial Conference of the African small ruminant research network, AICC, Arusha, Tanzania.

12. Bourdin P (1983) History, epidemiology and economic significance of PPR in Wes Africa and Nigeria in particular. In: Hill, D.H. (ed.), Pestes des petit ruminants (PPR) 
Abubakar MB (2017) Serological survey of antibodies against peste des petits ruminants virus (pprv) and Mycoplasma capricolum subsp. Capripneumoniae(mccp) among small ruminants in northern Cameroon

in sheep and goats. Proceedings of the International Workshop held at I.I.T.A., Ibadan, Nigeria, 1-11.

13. Awa DN, Njoya A, Ngo Tama AC (2000) Economics of prophylaxis against peste des petits ruminants and gastrointestinal helminthosis in small ruminants in north Cameroon. Trop Anim Health Prod 32: 391-403. [Crossref]

14. Diallo A, Gerrit JV, (2007) Genus Capripoxvirus, Poxviruses, Birkhäuser Advances in Infectious Diseases, Birkhäuser Basel. 167-181.

15. Martrenchar A, Bouchel D, Zoyem N (1995a) Isolation and experimental studies of Mycoplasma mycoidessubsp. Mycoides large colony and mycoplsmaovipneumoniaein goats in Nothern Cameroon. Small Ruminant Res 16: 179-184.

16. Martrenchar A, Zoyem N, Ngangnou A, Bouchel D, Ngo Tama AC, et al. (1995b) [Main infectious agents involved in the etiology of lung diseases of small ruminants in northern Cameroon]. Rev Elev Med Vet Pays Trop48: 133-137. [Crossref]

17. Martrenchar A, Bouchel D, Zoyem N, Thiaucourt F, Lambert M (1997) Risk factors responsible for the appearance of individual clinical signs in small ruminants in Northern Cameroon. Small Ruminant Res 26: 45-52.

18. MacOwan KJ, Minette JE (1976) A mycoplasma from acute contagious caprine pleuropneumonia in Kenya. Trop Anim Health Prod 8: 91-95. [Crossref]

19. Woubit S, Lorenzon S, Peyraud A, Manso-Silván L, Thiaucourt F (2004) A specific PCR for the identification of Mycoplasma capricolum subsp. capripneumoniae, the causative agent of contagious caprine pleuropneumonia (CCPP). Vet Microbiol104: 125-132. [Crossref]

20. Thiaucourt F, Bölske G, Libeau G, Le Goff C, Lefèvre PC (1994) The use of monoclonal antibodies in the diagnosis of contagious caprine pleuropneumonia (CCPP). Vet Microbiol41: 191-203. [Crossref]

21. Libeau G, Lancelot R, Colas F, Geurre L, Diallo A (1995) Development of a competitive ELISA for detecting antibodies to the peste des petits ruminants virus using a recombinant N protein. Res Vet Sci 58: 50-55.[Crossref]

22. Ferri NP, Dawson M (1988) Rountine application of enzyme linked immunosorbent assays in comparison with compliment fixation test for the diagnosis of FMD and swine vesicular stomatitis. Veterinary Microbiology 16: 201-209.
23. Martrenchar A, Zoyem N, Njoya A, Ngo Tama AC, Bouchel D, et al. (1999) Field study of a homologous vaccine against peste des petites ruminants in Northern Cameroon. Small ruminant res31: 277-280.

24. Batten CA, Banyard AC, King DP, Henstock MR, Edwards L, et al. (2011) A real time RT-PCR assay for the specific detection of Peste des petits ruminants virus. $J$ Virol Methods 171: 401-404. [Crossref]

25. El Harrak M, Touil N, Loutfi C, Hammouchi M, Parida S, et al. (2012) A reliable and reproducible experimental challenge model for peste des petits ruminants virus. $J$ ClinMicrobiol50: 3738-3740. [Crossref]

26. Durojaiye AO (1980) Brief notes on history, epizootiology and the economic importance of PPR in Nigeria. In: Hill, D.H. (ed.), Pestes des petit ruminants (PPR) in sheep and goats. Proceedings of the International workshop held at I.I.T.A., Ibadan, Nigeria, PP. 24-27.

27. Roeder PL, Abraham G, Kenfe G, Barrett T (1994) Peste des petits ruminants in Ethiopian goats. Trop Anim Health Prod 26: 69-73. [Crossref]

28. Hammouchi M, Loutfi C, Sebbar G, Touil N, Chaffai N, et al. (2012) Experimental infection of alpine goats with a Moroccan strain of Peste des Petits Ruminants virus (PPRV). Vet Microbiol 160: 240-244.[Crossref]

29. Desoutted LD (1994) Les pneumopathies des petites ruminants au Senegal: Etude seroepidemioloque. Thesed'Universite Paris, xii Val De Marne.Creteil.

30. Abegunde A (1980) Problems connected with TCRV vaccination of sheep and goats. In Hill, D.H. (ed.), Pestes des petit ruminants (PPR) in sheep and goats. Proceedings of the International Workshop held at I.I.T.A., Ibadan, Nigeria 79-81.

31. Taylor WP (1980) The use of TCRV and PPRV to confer immunity to PPR. In: Hill, D.H. (ed.), pestes des petit ruminants (PPR) in sheep and goats. Proceedings of the International workshop held at I.I.T.A, Ibadan, Nigeria, Pp. 72-73.

32. Baba SS, Fagbami AH, Ojeh CK (1999) Preliminary study on the use of solid-phase immunosorbent techniques for the rapid detection of wesselsbron virus IgM by haemagglutination-inhibition. Comp ImmunolMicrobiol Infect Dis 22: 71-79. [Crossref]

33. Bölske G, Johanson KE, Heinenen R, Panvuga PA, Twinnamasiko E (1996) Contagious caprine pleuropneumonia in Uganda and the isolation of Mycoplasma capricolumsubsp. capripneumoniae from goats and sheep. Vet Rec137: 594. [Crossref]

Copyright: $@ 2017$ Abubakar MB. This is an open-access article distributed under the terms of the Creative Commons Attribution License, which permits unrestricted use, distribution, and reproduction in any medium, provided the original author and source are credited. 